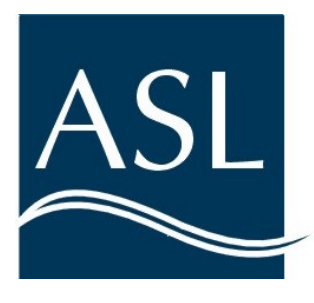

\title{
Inverted Echo Sounder on a Cabled Observatory
}

\author{
D. D. Lemon, R. A. Chave and M. R. Clarke, ASL Environmental Sciences Inc. \\ and \\ R. K. Dewey and P. Macoun, VENUS, University of Victoria
}

Presented at Oceans 2007, Vancouver

\begin{abstract}
High-frequency acoustic backscatter measurements have long been used as a method to detect zooplankton populations in the ocean. Ship-borne echo-sounders can map distributions over relatively large areas, but are not practical for following developments over long periods of time. Self-contained echo-sounders, either moored at depth looking upward, or mounted on surface buoys looking downward produce time series of acoustic backscatter which are a means for monitoring long-term behaviour of zooplankton populations. Combining these instruments with a cabled observatory allows this information to be acquired and monitored in near real-time, and thus to contribute to understanding and monitoring the state of one of the key components of coastal marine ecosystems. In this paper, we will discuss what we believe is the first operational example of an inverted echo sounder as part of a cabled seafloor observatory.
\end{abstract}

The first component of the VENUS seafloor observatory, in Saanich Inlet, British Columbia, began operation in February, 2006. The instrument platform is located $3 \mathrm{~km}$ from the Institute of Ocean Sciences dock, at 96m depth. A $200 \mathrm{kHz}$ upward looking echo sounder (Acoustic Water Column Profiler ${ }^{\mathrm{TM}}$, also known as the ZAP, for Zooplankton Acoustic Profiler) is located on the platform. The instrument collects a profile of acoustic backscatter strength throughout the water column once per second and transmits the data over the cable to the VENUS shore station. Power and control communications are also supplied over the cable. The techniques for command and control of the instrument over the VENUS VPN are described, as are the means for real-time display of the data using TCP/IP protocols. Continuous data retrieval and image posting to the VENUS website are performed automatically using the observatory's DMAS (Data Management and Archiving System). Echograms of acoustic backscattering strength as a function of depth and time are available on the web at hourly intervals, and as daily summaries. These plots, and the data files from which they are constructed, may be used for the detection if zooplankton, fish, near-surface bubbles and internal waves. Their evolution and development can be monitored in real time, and the archived data now provide a year-long record at the Saanich Inlet site.

At least two more of these instruments will be deployed at sites in Georgia Strait, when the next phase of the Venus observatory system is installed in October 2007. It is anticipated that the data from these locations will provide a valuable tool to further the understanding of the state of this coastal sea. A new generation of the AWCP is currently under development, which will provide greater A/D resolution and greater data storage for self-contained operation.

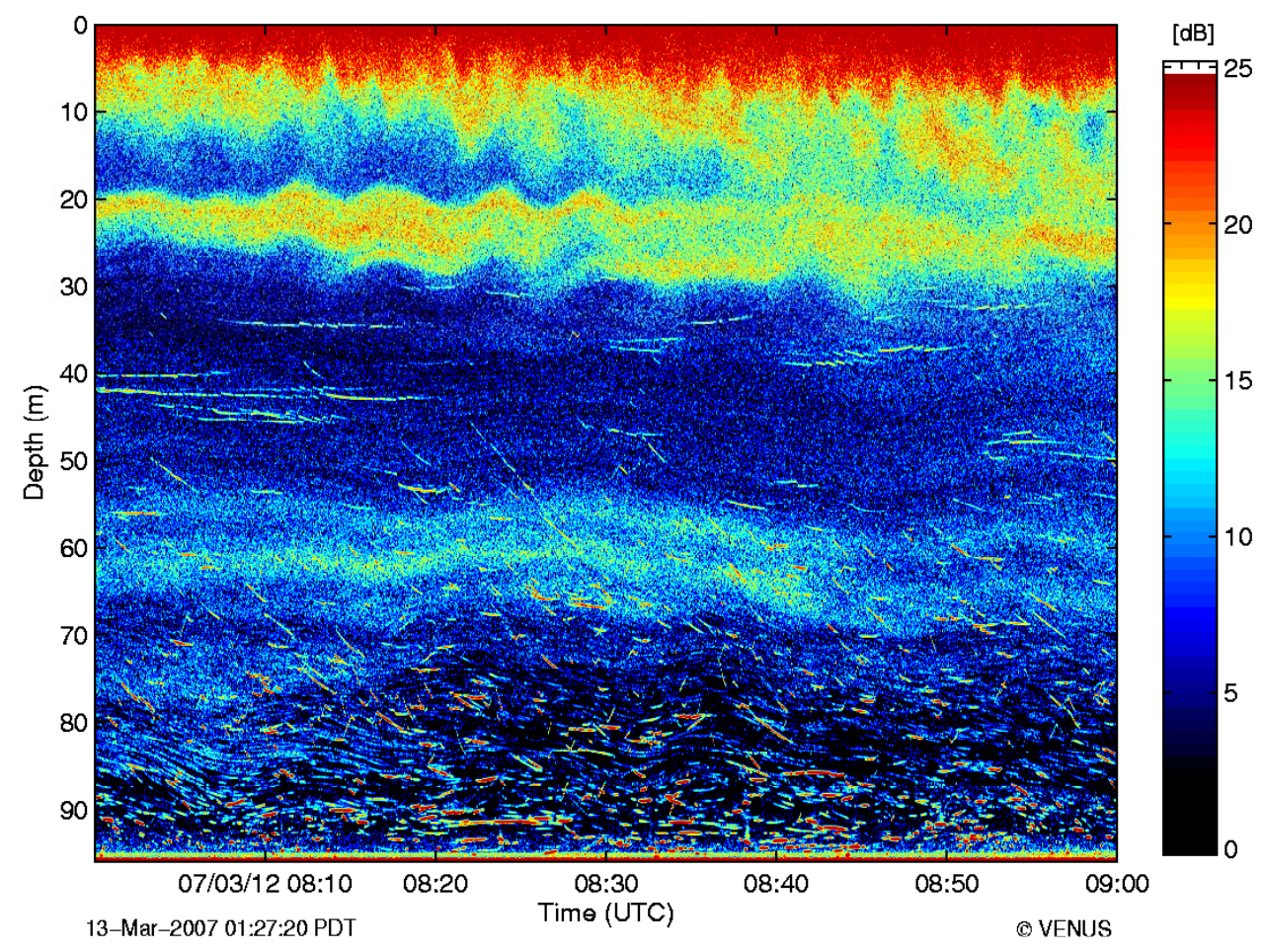

Fig. 1 Example hour of 200k Hz echo sounder data from the VENUS Observatory in Saanich Inlet, 13 March, 2007. 


\section{INTRODUCTION}

Knowledge of the processes governing the health of ocean ecosystems has always been limited by the difficulty of collecting long-term, comprehensive data in a vast, opaque medium. Biological oceanographers have had to attempt to understand oceanic food webs and species relationships from very limited data samples, in both space and time. Satellite sensors have overcome to a certain extent the spatial limitations in sampling, but only on the ocean surface. Obtaining data from beneath the ocean surface on populations of fish, zooplankton and other creatures can be done from ships using sampling nets and echo sounders. Highfrequency echo sounders mounted on ships can map the abundance and spatial distribution of zooplankton; using multiple frequencies, especially in conjunction with net sampling and optical detectors, can provide some information about the population's species composition [1]. While fairly large areas can be surveyed quickly in this fashion, it is impractical for monitoring changes continually over time.

Moored echo sounders can monitor backscatter from zooplankton over extended periods, with relatively high resolution both in time and depth. They can be mounted either on a surface buoy looking down, or subsurface, inverted to look upwards, either on the bottom or on a mid-water mooring [2,3,4]. These instruments are usually single-frequency devices, and therefore cannot separate the contributions to the backscatter signal from different species within a population. If high levels of turbulent microstructure are present, that can also contribute significantly to the backscatter $[5,6,7]$. In the absence of significant turbulence, if the population is dominated by a single species, or if the member species differ markedly in their behaviour, then moored single-frequency instruments are a very useful tool for monitoring long-term changes in zooplankton behaviour and abundance. As these instruments are self-contained, the duration, frequency and depth resolution of the sampling are constrained by power and data storage limitations, and the data are only available after the unit is recovered.

Incorporating an inverted echo sounder into a cabled observatory overcomes these limitations, and allows the information to be acquired and monitored in near real time, and with greater freedom in the choice of sampling interval and depth resolution. We believe that the $200 \mathrm{kHz}$ inverted echo sounder included in the instrument suite on the VENUS cable in Saanich Inlet, British Columbia, is the first operational example of this technique.

\section{INSTRUMENT DESCRIPTION}

The inverted echo sounder used at the VENUS observatory's Saanich Inlet site is a $200 \mathrm{kHz}$ Acoustic Water Column Profiler ${ }^{\mathrm{TM}}$ (AWCP), manufactured by ASL Environmental Sciences Inc. In its VENUS application, the instrument is also known as the ZAP, for Zooplankton Acoustic Profiler. Fig. 2 shows the instrument, as configured for the VENUS installation. Unlike the standard moored version of the AWCP, where the transducer is attached to one end cap, the ZAP's transducer is connected to the instrument body by a $3 \mathrm{~m}$ cable to allow the transducer to be oriented independently of the pressure case. A second cable

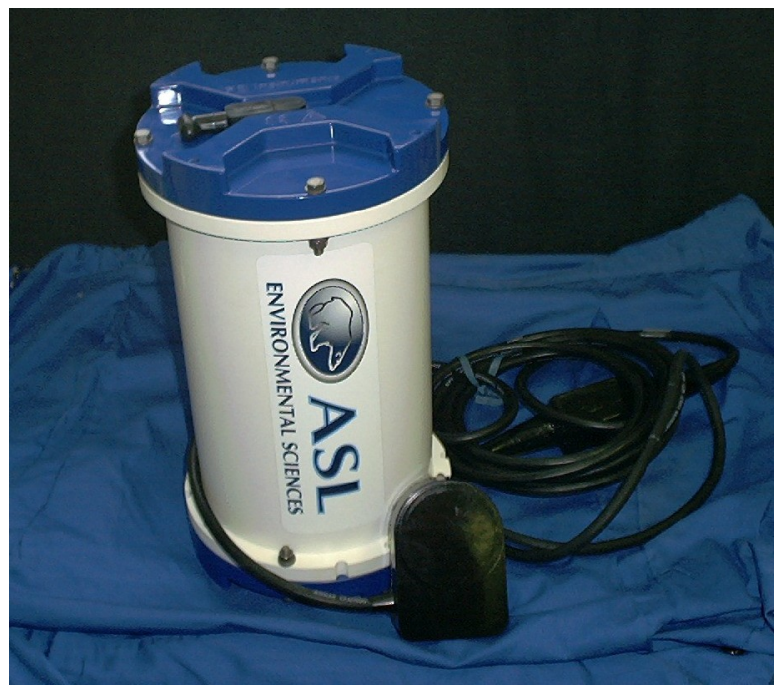

Fig. 2 The AWCP and transducer. provides the power and serial communications link to the VENUS observatory through a SIIM (Science Instrument Interface Module). Because of the indefinite duration of the deployment, the pressure case is plastic, to avoid periodically replacing anodes, as would be necessary with a metal housing; that is particularly important when there is a powered connection. The transducer has a $3 \mathrm{~dB}$ beamwidth of 8 degrees, with sidelobes at $-17 \mathrm{~dB}$ or less. The ZAP contains no data storage; all data are immediately transmitted through the cable to the VENUS Data Management and Archive System (DMAS). The instrument pings continuously at $1 \mathrm{~Hz}$; the transmissions have a source level of approximately $213 \mathrm{~dB}$ re $1 \mu \mathrm{Pa}$ at $1 \mathrm{~m}$ distance. The length of the transmitted pulse can be selected from 4 to $1020 \mu$ sec; the Saanich Inlet instrument is nominally set at $300 \mu \mathrm{sec}$.

Fig. 3 shows the signal path through the instrument up to the point at which the internal microprocessor acquires and formats the data for transmission over the serial port. After transmission, echoes arriving at the transducer pass through a preamplifier and bandpass filter, then through a time-varying gain (TVG) stage and an envelope detector to the A/D converter. The amplitude is sampled at $23.3 \mathrm{kHz}$ with 8 bit resolution to a selected maximum range of $200 \mathrm{~m}$ or less. The digitization 
rate provides range resolution of approximately $1 / 8$ metre. The time varying gain has an $80 \mathrm{~dB}$ range, and follows an approximate $20 \log \mathrm{R}+2 \alpha \mathrm{R}$ form, where $\alpha$ is the absorption coefficient for $200 \mathrm{kHz}$ sound in seawater and $\mathrm{R}$ is the range. The gain at which the TVG starts can be selected from one of 4 values, to allow some adjustment of the limited dynamic range of the 8-bit A/D. (1).

The counts, $\mathrm{N}$, output by the A/D range between 0 and 255 and are related to the volume backscatter strength $\mathrm{S}_{v}$ at range $\mathrm{R}$ by

$$
\mathrm{S}_{v}=20 \log \mathrm{N}-\mathrm{G}(2 \mathrm{R} / \mathrm{c})-\mathrm{OCV}-\mathrm{SL}+20 \log \mathrm{R}+2 \alpha \mathrm{R}-10 \log (1 / 2 \mathrm{c} \tau \Psi)
$$

where $\mathrm{c}$ is the speed of sound, $\tau$ is the transmitted pulse width, $\Psi$ is the equivalent solid angle of the transducer beam, $G(2 R / c)$ is the instrument time-varying gain function in $\mathrm{dB}$ and $\mathrm{OCV}$ is the transducer receiving response. If the time-varying gain function in the instrument exactly matches the spreading and absorption losses, inspection of (1) shows that the logarithm of $\mathrm{N}$ is directly proportional to $\mathrm{S}_{v}$. The TVG in the ZAP is an analogue circuit, and is based on an assumed speed of sound and absorption coefficient, and therefore does not usually exactly compensate for the spreading and absorption loss; correction for that difference is necessary to convert $\mathrm{N}$ to $\mathrm{S}_{v}$. The difference varies with range and can be $6 \mathrm{~dB}$ or greater, depending on the difference between the actual water properties and those assumed for the TVG. Using measured values for the TVG reduces the deviation from direct proportionality to approximately $3 \mathrm{~dB}$; calculation of $\mathrm{S}_{v}$ also requires precise calibration of the transducer beam pattern. Approximate values for $S_{v}$ can be calculated using the manufacturer's calibrations.

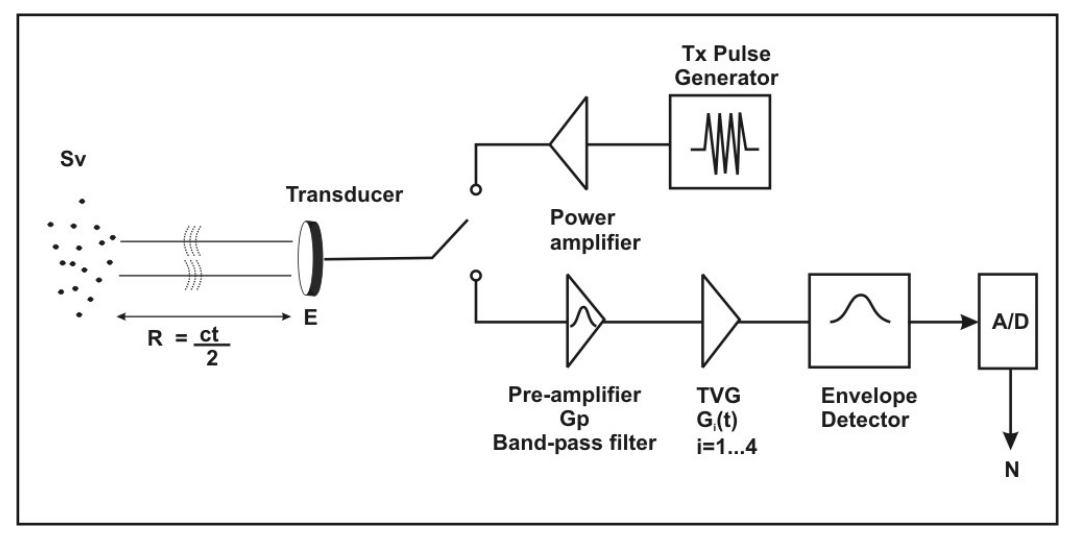

Fig. 3 Instrument signal path

The range in $\mathrm{S}_{v}$ that the ZAP can detect is determined by the dynamic range of the A/D, the TVG selected and the instrument's internal noise level. Increasing the overall gain lowers the maximum $S_{v}$ at which the A/D saturates and amplifies weaker returns, but also amplifies the self-noise. Lowering the overall gain allows detection of stronger returns without saturation, but weaker returns will then fall below the A/D's least bit level. The ZAP in Saanich Inlet uses TVG setting 2; at 50m range it can detect returns from $S_{v}$ between approximately -84 and $-40 \mathrm{~dB}$. At $100 \mathrm{~m}$ range, those limits are -73 to $-36 \mathrm{~dB}$.

\section{VENUS OBSERVATORY}

The VENUS cabled observatory is a permanent oceanographic research facility scheduled for completion by the end of 2007 [8]. It consists of two cabled arrays, the first located in Saanich Inlet, the second in the Strait of Georgia. The concept of the observatory is to utilize telecommunication technologies to connect oceanographic instrument systems to the research community via the Internet. The infrastructure includes a shore station that manages power and communications to the array, a back-bone fibre optic cable running from the shore station to observatory "Nodes", and instrument systems plugged into the Node. Wet-mate connectors at the Node provide 360 VDC power and 100 BaseT Ethernet communications to instruments. For legacy instruments, serial devices, and where many instruments need to be multiplexed, a Science Instrument Interface Module (SIIM) has been designed that provides individual control, monitoring, and communications to multiple instruments. The VENUS SIIM can provide either 360 VDC or 24 VDC power, and either 100/10 BaseT Ethernet or RS-232/422/485 serial communications. Via Internet back-haul connections, the shore station receives instructions and control from, and transmits data to, the University of Victoria, which houses both the Network Operations Centre (NOC) and the Data Management and Archive System (DMAS). DMAS provides both long-term data storage and distribution over the World Wide Web. Details of the observatory design are presented else where in these proceedings [8]. 
The ZAP was installed on the VENUS Instrument Platform (VIP) at $96 \mathrm{~m}$ depth at the entrance to Patricia Bay in Saanich Inlet (Fig. 4) in early 2006, and has been operating continuously since that time, with the only interruptions being those due to maintenance and failures in other parts of the VENUS system. The bottom-mounted VIP carries a number of instruments, including CTDs, oxygen and dissolved gas sensors, a transmissometer, an ADCP (at times) and the ZAP. The ZAP is mounted in one corner with its transducer pointing vertically upward (Fig. 5). Other systems connected to the observatory include a pan and tilt digital camera, and a hydrophone array for monitoring ambient sounds and marine mammal communications.

Real-time data are transmitted over the cable to the shore station, and thence to the DMAS on the University of Victoria campus. Presently, echograms showing the last 24 hours of data (colour-coded to show $\mathrm{N}$ in $\mathrm{dB}$ as a function of range and time) are available on the VENUS website (iwww.venus.uvic.ç) as a full day plot and as individual hourly plots. Galleries showing plots for any selected day are under development. ZAP data stored in the DMAS may be accessed as either tagged XML or raw HEX file formats. Access to observatory data is free, after a DMAS registration, which is used for tracking purposes only. Submitting a data request through the website for a specified period results in

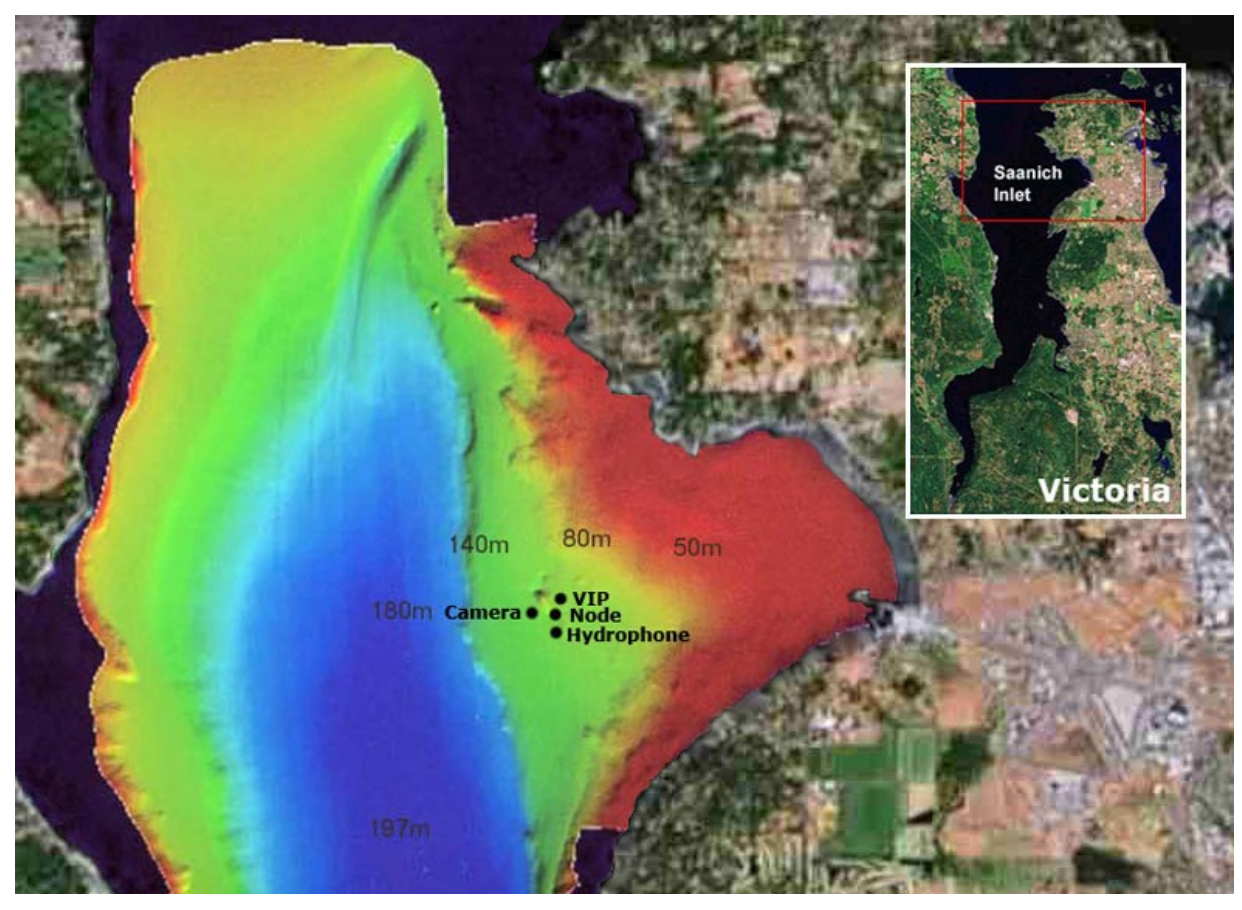
the files being posted to an ftp site, from which they may be downloaded.

Fig. 4 Location of the VENUS Observatory site in Saanich Inlet, BC. The ZAP is at the point marked VIP. Additional data formats and viewing options are under development.

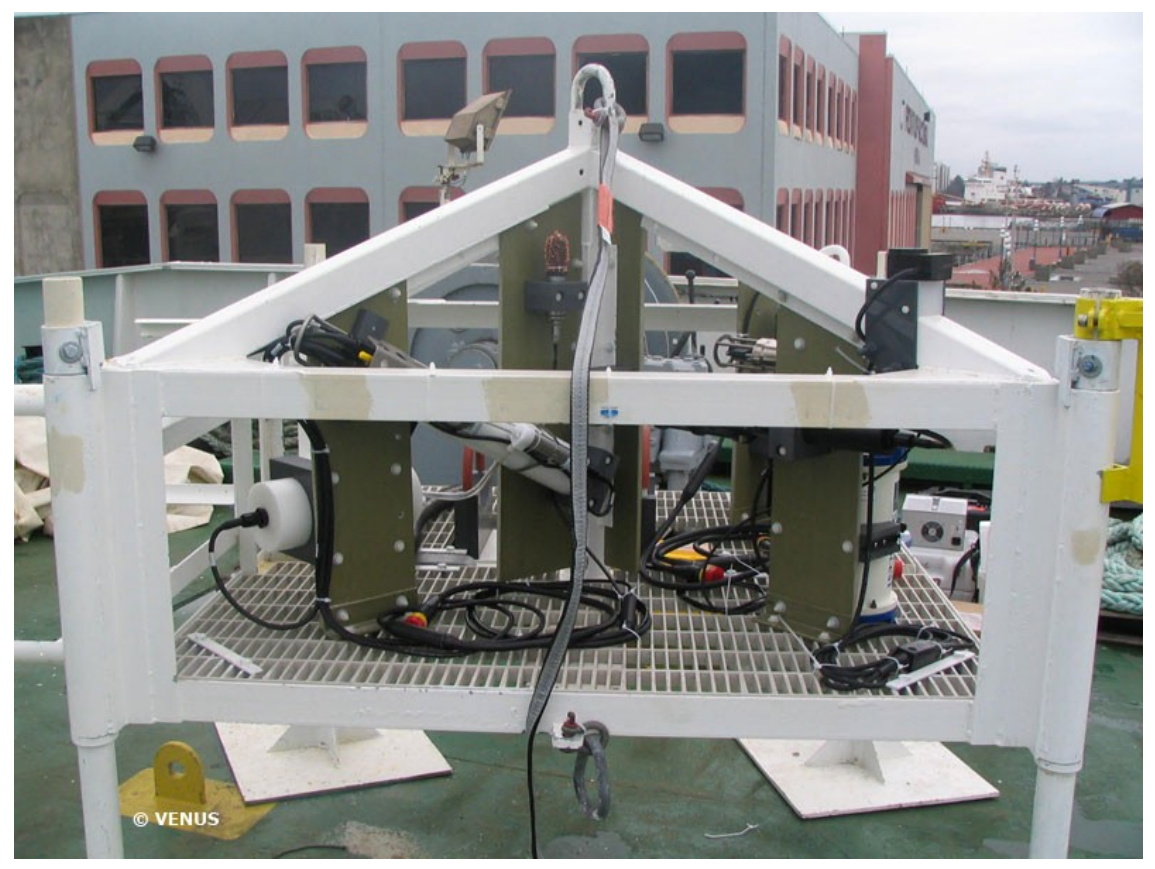

Fig. 5 The VENUS Instrument Platform; the ZAP transducer is on the right-hand upper bar, with the pressure case below. 


\section{DATA EXAMPLES}

The echograms from Saanich Inlet show a number of interesting phenomena. The most striking is the diurnal migration of a strong scattering layer (Fig. 6), which is most likely composed of euphausiids (E. Pacifica) [3], known to migrate to the surface at night. Other, non-migrating species such as copepods are also present in large numbers. Schools of small fish also show up clearly (Fig. 7) as do clouds of near-surface bubbles under rough, windy conditions. The scattering layers also make internal waves clearly visible. Variations over a season (Fig. 6) reveal a rich and diverse ecosystem. This extended time series offers abundant opportunity for detailed investigation of these and other phenomena.
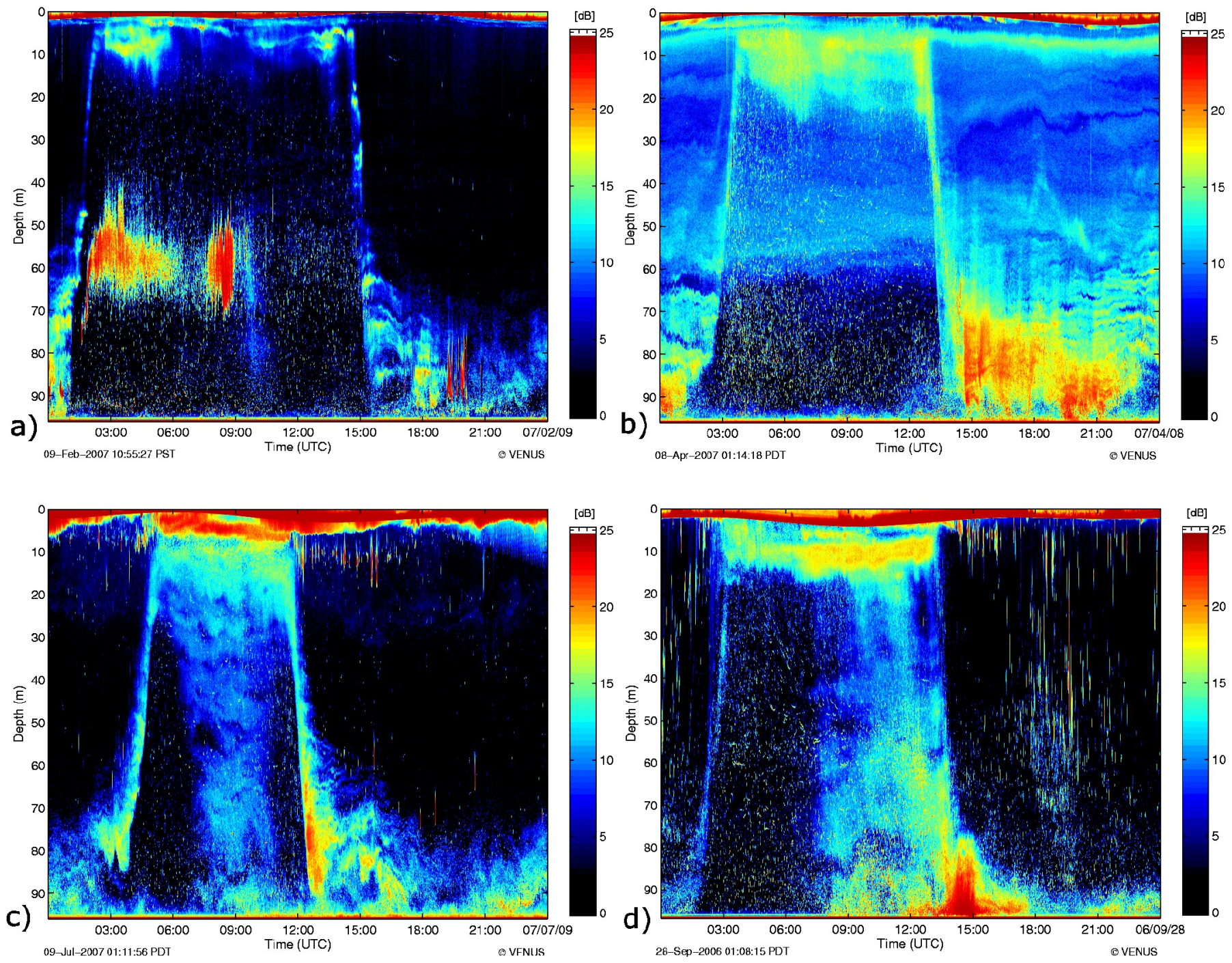

Fig. 6 Example 24 hour ZAP images from the VENUS Observatory in Saanich Inlet. The four panels are representative of the various seasons: a) Winter: 9 February 2007, b) Spring: 8 April, 2007, c) Summer: 9 July, 2007, d) Fall: 28 September, 2006. Diel zooplankton migration is a dominant signal, but other seasonal and temporal variations are evident.

The VENUS observatory has several co-located active acoustic systems, including the ZAP and an Acoustic Doppler Current Profiler. Although the carrier frequencies are often distinct (i.e. 200 and $300 \mathrm{kHz}$ ), the broadband nature of the pulsed and coded signals means that special care must be taken to avoid interference. Instrument control and data acquisition protocols are under development that will allow for the accurate tracking of instrument clocks, the synchronization of transmit and receive signals, and the interleaving of distinct sonar systems. 


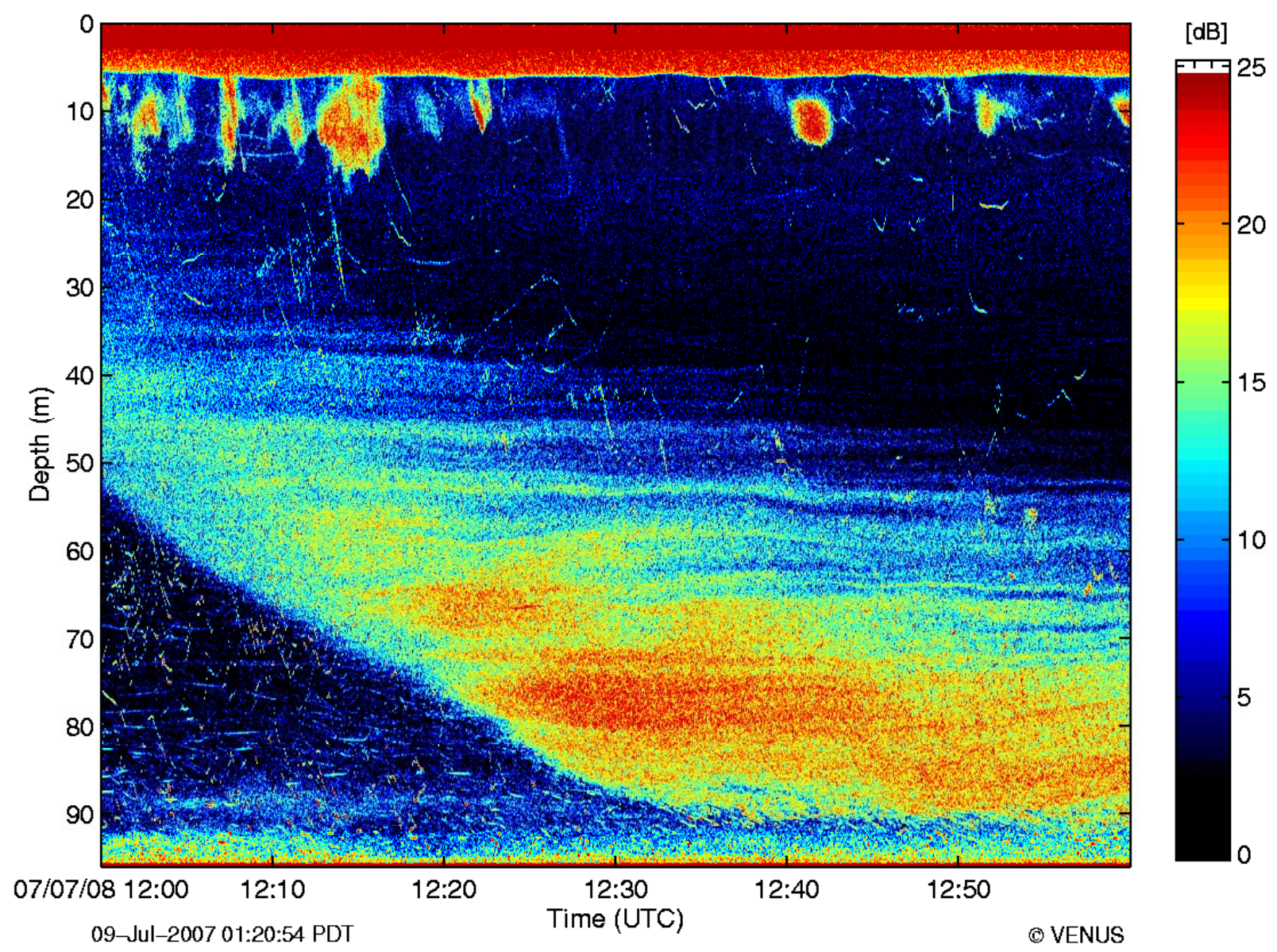

Fig. 7 An example of one hour of ZAP data from the VENUS observatory in Saanich Inlet, 7 July, 2007. The ping interval is 1 second, resulting in 3600 profiles per hour. Zooplankton are seen during their dawn descent, along with individual fish (small streaks) and schools of bait fish near the surface. A strong echo return from the water surface also tracks the tidal elevations.

\section{FUTURE PLANS}

The cable for the Strait of Georgia VENUS array was successfully deployed in May from the Cable Ship Wave Venture (Fig. 8). Two observatory Nodes will be located at the 300 and $170 \mathrm{~m}$ isobaths, south west of the Fraser River's mouth. An extension cable will also run up towards the river mouth to support a suite of instruments dedicated to monitoring sediment transport processes from the river. Preparations for the deployment of the nodes and instrumentation this fall (October 2007) are presently under way. Two more ZAP instruments will be deployed at the Node locations shown in Fig. 8, and are expected to provide valuable information about the state of the coastal sea.

A new generation of the AWCP, the AWCP-5, is also under development and due for release later this year. The AWCP-5 incorporates a more powerful processor, greatly expanded data storage for self-contained operation (up to 8 and eventually 16 GBytes) and 16-bit digitization. The greater dynamic range in the digitizer eliminates the need for selectable gain ranges, and adds $9 \mathrm{~dB}$ to the detectable volume backscatter range obtained with the existing instrument design.

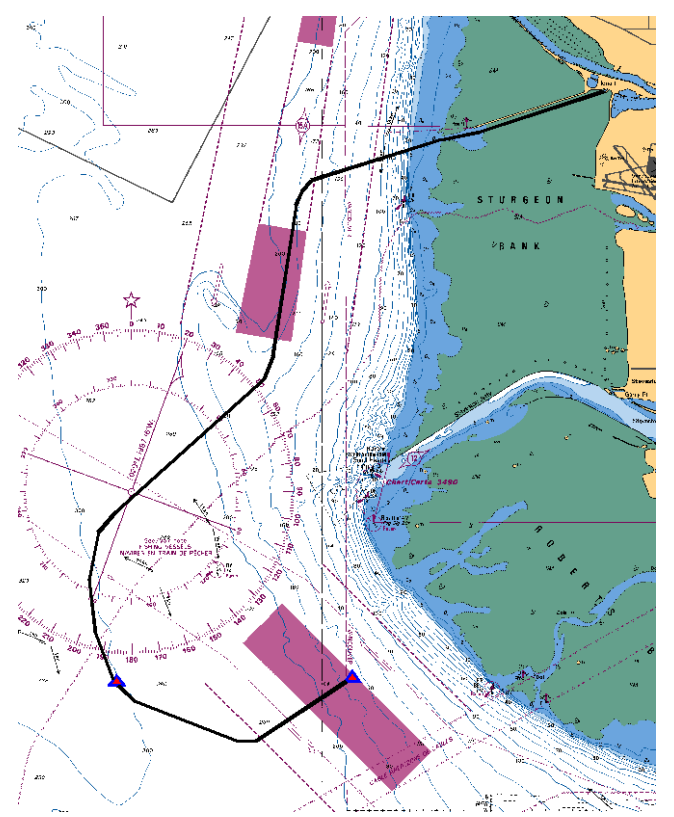

Fig. 8 VENUS Observatory cable in the Strait of Georgia. Blue triangles indicate the Node locations. 
Funding for the VENUS project was provided by the Canadian Foundation for Innovation and the British Columbia Knowledge Development Fund. In-kind contributions also came from the University of Victoria, NEPTUNE Canada, Camosun College, National Research Council, Natural Resources Canada, Fisheries and Oceans Canada, Department of National Defense, 2WE Associates Consulting Ltd., Alcatel Submarine Networks, ASL Environmental Sciences Inc., Barrodale Computing Services, Jet Propulsion Lab; NASA, Ocean Design Inc., Pro-Oceanus, Sun Microsystems. Assistance with instrument configuration and data displays was provided by Paul Macoun and Jaklyn Vervynck, respectively.

\section{REFERENCES}

[1] M. V. Trevorrow, D. L. Mackas and M. C. Benfield, "Comparison of multifrequency acoustic and in situ measurements of zooplankton abundances in Knight Inlet, British Columbia,” J. Acoust. Soc. Amer., vol. 117 (6), pp. 3574-3588, June 2005.

[2] D. D. Lemon, J. F. R Gower, M. V. Trevorrow and M. R. Clarke, "The acoustic water column profiler: a tool for long-term monitoring of zooplankton populations," Proc. MTS/IEEE International Conf. Oceans 2001, vol. 3, pp. 1904-1909, November, 2001.

[3] M. V. Trevorrow, “ The use of moored inverted echo sounders for monitoring meso-zooplankton and fish near the ocean surface," Can. J. Fish. Aquat. Sci., vol. 62, pp. 1004-1018, May 2005.

[4] A. S. Brierly, R. A. Saunders, D. G. Bone, E. J. Murphy, P. Enderlein, S. G. Conti and D. A. Demer, "Use of moored acoustic instruments to measure shortterm variability in abundance of Antarctic krill," Limn. Oceanogr.: Methods, vol. 4, pp. 18-29, February 2006.

[5] M. V. Trevorrow, “Observations of internal solitary waves near the Oregon coast with an inverted echo sounder,” J. Geophys. Res. vol. 103 (C4), pp. 7671$7680,1998$.

[6] T. Ross and R. Lueck, "Sound scattering from oceanic turbulence,” Geophys. Res. Lett., vol. 30, pp.1344-1347, 2003.

[7] E. Kunze, J. F. Dower, I. Beveridge, R. Dewey and K. P. Bartlett, “Observations of biologically generated turbulence in a coastal inlet,” Science, vol. 313, pp. 1768-1770, September 2006.

[8] R. Dewey, V. Tunnicliffe, and A. Round, "The VENUS Cabled Observatory: An Underview of Engineering Meets Science.”, Proc. MTS/IEEE International Conf. Oceans 2007, October, 2007. 
